\title{
Conocimientos respecto a la identificación y manejo del trastorno depresivo mayor en internos y residentes de medicina de la Universidad Peruana Cayetano Heredia en el año 2011.
}

Knowledge about identification and management of major depressive disorder in interns and medical residents of the Universidad Peruana Cayetano Heredia in the year 2011.

María José Inurritegui-Cattaneoㅁ, Elisa Juárez-Chávez¹, Johann M. Vega-Dienstmaier²

\section{RESUMEN}

Objetivos: Explorar los conocimientos de los internos de medicina y residentes de especialidades médicas de la Universidad Peruana Cayetano Heredia acerca del diagnóstico y tratamiento del trastorno depresivo mayor. Metodología: Se realizó un estudio transversal durante enero y febrero de 2011. Se elaboró un cuestionario para evaluar conocimientos acerca del diagnóstico y manejo de depresión mayor, el cual fue validado mediante revisión de jueces y luego se hizo un piloto con ex-alumnos de medicina egresados el año 2010. Posteriormente se aplicaron los cuestionarios a 36 médicos residentes de medicina y 82 internos. Resultados: El 91,5\% de los participantes realizó una correcta impresión diagnóstica de depresión mayor, el 97\% consideró que sí debía darse tratamiento farmacológico, pero sólo el 48,8\% de internos y 33,3\% de residentes prescribió una terapéutica correcta. Los antidepresivos mencionados como primera línea fueron los inhibidores selectivos de la recaptación de serotonina $(91,2 \%)$, siendo el de mayor preferencia, la sertralina (65,4\%), seguida de la fluoxetina (24\%). Más del $90 \%$ de los participantes sugirió adicionar un tratamiento no farmacológico y una interconsulta a otro servicio. En relación al conocimiento sobre cómo elegir el antidepresivo, vemos que el promedio de respuestas acertadas por los internos fue de 3,8 $(\mathrm{DE}=1,99)$ mientras que entre los residentes, el promedio de respuestas correctas fue de 3,08 $(\mathrm{DE}=1,56)$. Conclusiones: No hay mayor dificultad para realizar un correcto diagnóstico de depresión mayor, tanto en internos como en residentes, sin embargo hay deficiencias para la prescripción de un tratamiento completo adecuado. (Rev Neuropsiquiatr 2011;74:200-208).

PALABRAS CLAVE: trastorno depresivo mayor, antidepresivos, médicos residentes, internos de medicina.

Médico. Universidad Peruana Cayetano Heredia. Lima, Perú.

2 Psiquiatra. Sección de Psiquiatría y Salud Mental; Universidad Peruana Cayetano Heredia. Servicio de Neuro-

Psiquiatría; Hospital Nacional Cayetano Heredia. Lima, Perú. 


\section{SUMMARY}

Objectives: To explore the knowledge of the interns and medical residents of the Universidad Peruana Cayetano Heredia about the diagnosis and treatment of major depressive disorder. Methods: A cross-sectional study was made on January and February 2011. A questionnaire was developed to evaluate the knowledge about the diagnosis and treatment of major depression; this was validated by means of experts revision and then, a pilot was fulfilled by former interns. After this process was completed, the questionnaire was taken by 36 medical residents and 82 interns. Results: A total of $91.5 \%$ of participants made a correct diagnosis of major depression, $97 \%$ considered necessary to give pharmacologic treatment, but only $48.8 \%$ of interns and $33.3 \%$ of residents gave an adequate prescription. The antidepressants given as a first line treatment were selective serotonin reuptake inhibitors, being the most prescribed sertraline $(65.4 \%)$ followed by fluoxetine $(24 \%)$. Over $90 \%$ of participants added a nonpharmacologic treatment and a consultation to another service. About the knowledge on how to choose an antidepressant, we found that the average of right answers among the interns was $3.8(\mathrm{SD}=1.99)$ while among the residents was $3.08(\mathrm{SD}=1.56)$. Conclusions: There is no difficulty in making an adequate diagnosis of a major depression case, neither in interns nor residents, but there is a lack of knowledge about the right treatment. (Rev Neuropsiquiatr 2011;74:200-208).

KEYWORDS: major depressive disorder, antidepressants, medical residents, medical interns.

\section{INTRODUCCIÓN}

Los trastornos depresivos son altamente prevalentes a nivel mundial, llegando a ser un importante problema de salud pública. Además no sólo afectan la calidad de vida, sino que también se asocian con una discapacidad funcional mayor que la generada por otras enfermedades, tales como la diabetes o la hipertensión (1).

Los trastornos depresivos unipolares son la primera causa de pérdida de años de vida saludable (PAVS) en el mundo en ambos sexos (8,3\% del total de PAVS en varones y $13,4 \%$ del total de PAVS en mujeres) (2).

El Perú no es ajeno a esta realidad. En el Estudio Epidemiológico Metropolitano de Salud Mental, realizado en el 2002 por el Instituto Nacional de Salud Mental "Honorio Delgado-Hideyo Noguchi" (INSM), se encontró que en la población adulta, 2 de cada 10 personas padecen de algún trastorno psiquiátrico, siendo los trastornos depresivos y ansiosos los más frecuentes. La prevalencia también es alta en adolescentes, población en la que se halló que 1 de cada 12 sufre algún trastorno depresivo y una cantidad similar ha tenido pensamientos suicidas (3).

Estas cifras no sólo se centran en Lima, donde la prevalencia de vida de depresión mayor es $18,3 \%$, también en la Sierra y la Selva las cifras son altas, con prevalencias de $16,2 \%$ y $21,4 \%$ respectivamente $(4,5)$.
Sin embargo, a pesar de las altas tasas de depresión, la proporción de personas que reciben tratamiento es baja, $25 \%$ en Lima y $13 \%$ en la Sierra y Selva (3-5).

La mayoría de personas con depresión que buscan ayuda, no lo hacen con un psiquiatra, sino con médicos generales o de familia (6). Por lo tanto, es muchas veces la visita al médico no psiquiatra la única oportunidad para identificar a estos pacientes e iniciarles un tratamiento adecuado.

En un estudio publicado en el año 2005, donde se encuestó a médicos asistentes y residentes de 3 hospitales de Lima acerca de la consulta ambulatoria no-psiquiátrica, el 93,1\% refiere haber realizado el diagnóstico de depresión en algún momento (7). Asimismo, en otra publicación sobre el mismo estudio, sólo un $32,3 \%$ y $16,9 \%$ de médicos generales pudo listar suficientes síntomas de depresión como para tener la posibilidad de hacer un diagnóstico en base a los criterios del CIE y el DSM, respectivamente (8).

Otra razón importante por la cual la depresión mayor se podría encontrar subdiagnosticada, es porque mayormente se presenta de manera atípica o acompañada de sintomatología somática (9).

Por lo expuesto, es muy importante que los médicos generales sepan cómo identificar y tengan la capacidad de tratar un trastorno tan prevalente como la depresión mayor, lo cual es reconocido por muchos autores (10). 
Actualmente se cuenta con guías de manejo para depresión dirigidas tanto a médicos especialistas como médicos generales, las cuales indican de manera clara y concisa los criterios para el diagnóstico de los trastornos depresivos entre otros trastornos psiquiátricos, así como los distintos fármacos que se emplean en el tratamiento. Asimismo, en el Perú el Ministerio de Salud ha hecho una Guía de Práctica Clínica en Depresión (11).

Consideramos de suma importancia conocer la capacidad de los internos como futuros médicos generales y de los residentes de especialidades médicas, para identificar a un paciente con trastorno depresivo mayor e indicar un tratamiento adecuado y en caso de hallar deficiencias poder implementar herramientas para su mejora.

\section{MATERIALES Y MÉTODOS}

Se realizó un estudio descriptivo transversal durante enero y febrero del 2011. Se utilizó una encuesta para conocimientos sobre depresión mayor, la cual se creó en base a los criterios diagnósticos del DSM-IV TR y la CIE 10. Dicha encuesta constaba de un caso clínico típico, con la suficiente información como para determinar el diagnóstico, y a partir de él se formularon preguntas abiertas y de opción múltiple. Este cuestionario fue enviado a cinco jueces especialistas (psiquiatras), quienes sugirieron algunos cambios relacionados a la manera en que se formularon las preguntas y concluyeron que el caso clínico era útil para evaluar conocimientos sobre depresión mayor. Posterior a ello, se realizó un piloto, aplicando la encuesta a 10 alumnos egresados del año 2010 de la Universidad Peruana Cayetano Heredia (UPCH), que señalaron que todas las preguntas se entendían claramente y que las preguntas de opción múltiple a su parecer tenían un grado de dificultad alto. El tiempo promedio de duración para contestar la encuesta fue de 10 minutos.

La encuesta consta de dos partes: la primera recogió datos generales, tanto para los residentes como para los internos; tales como: edad, sexo, rotaciones que realizó durante el externado (para internos) y año de residencia y año en el cual culminó sus estudios de pregrado (para residentes). A continuación mostramos el caso clínico luego del cual se formularon 4 preguntas abiertas sobre diagnóstico, tratamiento farmacológico, tratamiento no farmacológico y derivación del paciente a otro servicio.

\section{Caso clínico}

Ana tiene 23 años, estaba estudiando en una academia, pero la dejó para trabajar en el puesto de ropa que tienen sus padres. Desde hace 4 meses, Ana comenzó a sentirse triste por momentos y luego de un mes estaba triste todo el día. Cuando sus padres no estaban en casa se iba a llorar a su cuarto, no tenía ganas de hacer sus cosas, se sentía cansada y se había equivocado varias veces cuando tenía que dar el vuelto a un cliente porque no se podía concentrar. Con el tiempo, Ana dejó de reunirse con sus amigas, ya no las visita, pasa la mayor parte del tiempo en su casa, le ha pedido a su madre que le dé permiso y se encargue del puesto de ropa porque ella se siente cansada. Por otro lado, le ha disminuido el apetito, ha bajado de peso, no puede dormir bien porque se levanta varias veces en la noche y le cuesta volver a conciliar el sueño. A partir de las 4 de la mañana ya no puede volver a dormir. Hace 2 semanas le dice a su madre que está sufriendo mucho y por momentos quisiera dormirse y no despertar. Sus padres están muy preocupados por todo lo que le está pasando a Ana y la quieren llevar a un especialista porque además últimamente se queja mucho de dolores de cabeza.

A continuación mostramos la segunda parte en la cual se evaluaron los conocimientos sobre la elección correcta de los antidepresivos, formulándose 10 preguntas de opción múltiple:

\section{Preguntas de opción múltiple (sólo marque una)}

1.- Si la paciente no quisiera aumentar de peso, ¿Cuál de los siguientes escogería?
a) Amitriptilina
b) Mirtazapina
c) Bupropion (respuesta correcta)
d) Sertralina

2.- Si la paciente tuviera un problema cardiaco (defecto de conducción), ¿Cuál de los siguientes no le daría?

a) Inhibidores selectivos de la recaptación de Serotonina

b) Inhibidores de la Monoaminooxidasa

c) Triciclicos (respuesta correcta)

d) Inhibidores de la recaptación de serotonina y noradrenalina

3.- Si la paciente sufriera de fibromialgia, ¿Cuál de los siguientes escogería?
a) Nortriptilina
b) Amitriptilina (respuesta correcta)
c) Venlafaxina
d) Fluoxetina 
4.- Si la paciente tuviera epilepsia, ¿Cuál de los siguientes preferiría?
a) Bupropion
b) Sertralina (respuesta correcta)
c) Clomipramina
d) Maprotilina

5.- Si la paciente tuviera Diabetes Mellitus, ¿Cuál sería el fármaco menos recomendado?
a) Fluoxetina
b) Citalopram
c) Bupropion
d) Amitriptilina (respuesta correcta)

6.- Si la paciente no desea tener efectos adversos en su vida sexual, ¿Cuál escogería?
a) Duloxetina
b) Sertralina
c) Paroxetina
d) Bupropion (respuesta correcta)

7.- Si la paciente tuviera retención urinaria, ¿Cuál de los siguientes preferiría?
a) Clomipramina
b) Paroxetina
c) Fluoxetina (respuesta correcta)
d) Amitriptilina

8.- Si la paciente tuviera glaucoma de ángulo cerrado, ¿Cuál sería el menos recomendable?
a) Fluvoxamina
b) Escitalopram
c) Amitriptilina (respuesta correcta)
d) Duloxetina

9.- Si la paciente desea un fármaco con el menor efecto sedante o hipnótico, ¿Cuál escogería?
a) Bupropion (respuesta correcta)
b) Fluvoxamina
c) Mirtazapina
d) Clomipramina

10.- $\mathrm{Si}$ la paciente además tuviera trastorno de ansiedad generalizada, ¿Qué antidepresivo no utilizaría?
a) Paroxetina
b) Bupropion (respuesta correcta)
c) Sertralina
d) Venlafaxina

Luego de ser respondida la primera parte inmediatamente se guardó y se procedió a entregar las preguntas de opción múltiple, evitando de esta manera posibles sesgos. Al final del procedimiento se adjuntaron ambas partes y se guardaron en un sobre cerrado.

El sustento a todas las respuestas de las preguntas de la encuesta se encuentran en las guías sobre manejo de depresión mayor de la American Psychiatric
Association (APA) del 2010, de la World Federation of Societies of Biological Psychiatry (WFSBP) del 2007 y de la Canadian Psychiatric Association junto con la Canadian Network for Mood and Anxiety Treatments del 2001 (13-16).

Las respuestas consideradas correctas para impresión diagnóstica fueron: "depresión”, "depresión mayor", "trastorno depresivo mayor" y "episodio depresivo mayor". Se consideró como correcto el tiempo total de tratamiento si estaba entre 6 meses y 2 años. Se consideró "terapéutica completa correcta" si el fármaco, la dosis y la duración del tratamiento fueron acertados. Como terapias no farmacológicas con nivel de evidencia I se consideraron las psicoterapias cognitivo-conductual, conductual e interpersonal (15).

Luego se inició la recolección de la información. Se ubicó al total de los internos, los cuales se encontraban realizando sus rotaciones en el Hospital Nacional Cayetano Heredia ( $\mathrm{HNCH})$, Hospital Arzobispo Loayza, Hospital de Chancay y Hospital de Huaral. Asimismo se ubicó a los residentes de la UPCH que realizan sus actividades en el HNCH. Se les solicitó su colaboración para completar la encuesta y se cuantificó el número de internos y residentes que rechazaron el procedimiento. Finalmente se guardó dicha información en un sobre para mantener la confidencialidad del participante, asegurando su anonimato. La participación fue voluntaria y el consentimiento informado fue verbal. El estudio fue presentado al Comité de Ética de la UPCH y tuvo exoneración de revisión por dicha entidad.

Se obtuvo el porcentaje de internos y residentes que reconocieron de forma correcta el diagnóstico y que plantearon un tratamiento psicofarmacológico adecuado, considerando el medicamento, la dosis y el tiempo de prescripción correctos. Asimismo se determinó el porcentaje de encuestados que sugirieron un tratamiento no farmacológico adecuado y que derivarían al paciente a Psiquiatría, Psicología u otros servicios. Se describió la proporción de individuos que contestaron correctamente cada pregunta del cuestionario de opción múltiple respecto a conocimientos sobre la elección del fármaco y se contabilizó el total de respuestas correctas para cada sujeto.

Además se realizaron comparaciones utilizando la prueba de Chi cuadrado o de Fisher para examinar diferencias de proporciones; la prueba de KormogorovSmirnov para evaluar si la distribución de las variables numéricas se aleja de la curva normal; y la Prueba To 
la de Mann-Whitney para examinar si hay diferencias entre grupos respecto a variables numéricas, según corresponda.

\section{RESULTADOS}

La población total de internos fue de 96 alumnos, de los cuales respondieron a la encuesta el $85,41 \%$ $(\mathrm{n}=82)$. El total de residentes fue de 65 médicos, de los cuales respondieron el 55,38\% $(n=36)$. No se logró ubicar al resto de internos y residentes. De los participantes, el 43,2\% ( $n=51)$ fueron del sexo femenino. La edad promedio de los internos fue 23,5 \pm
1,6 años y la de los residentes $29,7 \pm 3,3$ años. Para los residentes, el tiempo promedio de egresado fue 5,89 $\pm 2,7$ años. El 51,2\% $(\mathrm{n}=42)$ de los internos realizó su rotación de psiquiatría de externado en el INSM y el $48,8 \%(\mathrm{n}=40)$ en el $\mathrm{HNCH}$.

En la tabla 1 se describen las respuestas de los sujetos evaluados relacionadas con sus conocimientos acerca del diagnóstico y manejo que darían al caso típico de depresión mayor presentado. Aquí observamos que el $91,5 \%(n=108)$ tuvo una impresión diagnóstica correcta, mientras que el $8,5 \%(\mathrm{n}=10)$ consideró otros diagnósticos, tales como: trastorno de adaptación y

Tabla 1. Conocimientos acerca del diagnóstico y manejo farmacológico y no farmacológico ante un caso de depresión mayor entre internos y residentes de medicina.

\begin{tabular}{|c|c|c|}
\hline & $\begin{array}{c}\text { INTERNOS }(\mathrm{n}=82) \\
\%\end{array}$ & $\begin{array}{c}\text { RESIDENTES }(\mathrm{n}=36) \\
\%\end{array}$ \\
\hline Consideró diagnóstico correcto & $93,9 \%(n=77)$ & $86,1 \%(n=31)$ \\
\hline $\begin{array}{l}\text { Consideró dar tratamiento } \\
\text { farmacológico }\end{array}$ & $97,6 \% \quad(n=80)$ & $97,2 \% \quad(n=35)$ \\
\hline Consideró ISRS como primera línea & $90,2 \% \quad(n=74)$ & $83,3 \%(n=30)$ \\
\hline $\begin{array}{l}\text { Consideró terapéutica completa } \\
\text { correcta } *\end{array}$ & $48,8 \%(n=40)$ & $33,3 \%(n=12)$ \\
\hline $\begin{array}{l}\text { Consideró dar tratamiento no } \\
\text { farmacológico }\end{array}$ & $98,8 \%(n=81)$ & $94,4 \% \quad(n=34)$ \\
\hline Consideró Psicoterapia en general & $31,7 \%(n=26)$ & $38,8 \%(n=14)$ \\
\hline Consideró Psicoterapia de apoyo & $3,6 \%(\mathrm{n}=3)$ & $30,5 \%(n=11)$ \\
\hline $\begin{array}{l}\text { Mencionó medidas sin evidencia sólida } \\
\text { de eficacia** }\end{array}$ & $43,9 \% \quad(n=36)$ & $13,8 \% \quad(n=5)$ \\
\hline $\begin{array}{l}\text { Consideró terapia con nivel de } \\
\text { evidencia I (TCC-TC) }\end{array}$ & $14,6 \%(n=12)$ & $8,3 \%(n=3)$ \\
\hline $\begin{array}{l}\text { No especifica ninguna medida no } \\
\text { farmacológica }\end{array}$ & $4,8 \%(n=4)$ & $2,7 \%(\mathrm{n}=1)$ \\
\hline Consideró enviar interconsulta & $80,5 \%(n=66)$ & $94,4 \% \quad(n=34)$ \\
\hline $\begin{array}{l}\text { Consideró interconsulta al Servicio de } \\
\text { Psiquiatría }\end{array}$ & $37,8 \%(n=31)$ & $50,0 \% \quad(n=18)$ \\
\hline $\begin{array}{l}\text { Consideró inteconsulta al Servicio de } \\
\text { Psicología }\end{array}$ & $12,2 \%(n=10)$ & $2,7 \%(n=1)$ \\
\hline $\begin{array}{l}\text { Consideró interconsulta al Servicio de } \\
\text { Psiquiatría y Psicología }\end{array}$ & $12,2 \%(n=10)$ & $22,2 \% \quad(n=8)$ \\
\hline $\begin{array}{l}\text { Consideró interconsulta al Servicio de } \\
\text { Psiquiatría y otro }\end{array}$ & $3,65 \% \quad(n=3)$ & $11,1 \% \quad(n=4)$ \\
\hline $\begin{array}{l}\text { Consideró interconsulta sólo a otro } \\
\text { servicio (Neuro logía, Medicina Interna) }\end{array}$ & $14,6 \%(n=12)$ & $8,3 \%(n=3)$ \\
\hline
\end{tabular}

*En cuanto a fármaco, dosis y duración del tratamiento

** Por ejemplo: "apoyo familiar", "salir a pasear", "hacer ejercicios", etc.

TCC: Terapia cognitivo-conductual

TC: Terapia conductual. 
episodio psicótico. En cuanto al tratamiento, el 97,6\% $(n=80)$ de los internos y $97,2 \%(n=35)$ de residentes consideró que debía darse algún tratamiento farmacológico. Sólo 3 personas consideraron que no era necesario hacerlo porque hicieron otro diagnóstico.

De los participantes que sí darían tratamiento farmacológico, se vio que el $91,2 \%(n=104)$, eligieron como fármaco de primera línea un inhibidor selectivo de la recaptación de serotonina (ISRS). Además se observó que dentro del grupo de los ISRS, el de mayor preferencia fue sertralina tanto en internos como en residentes con un $65,4 \%(n=68)$, seguido por fluoxetina con un $24 \%(n=25)$. En cuanto a la terapéutica indicada (fármaco, dosis y duración total del tratamiento), se encontró una tendencia $(\mathrm{p}=0,12$, Chi2) de los internos a prescribir un tratamiento completo correcto respecto de los residentes, con un $48,8 \%(\mathrm{n}=40)$ y $33,3 \%(\mathrm{n}=12)$, respectivamente.

Un 62,5\% $(\mathrm{n}=25)$ de los internos que realizaron su rotación de psiquiatría en el $\mathrm{HNCH}$ plantearon un tratamiento completo correcto, mientras que para los que rotaron en el INSM, esta cifra correspondió a $35,7 \%(\mathrm{n}=15)(\mathrm{p}=0,015$, Chi2 $)$.
En cuanto a la terapia no farmacológica, podemos observar que, entre los internos, el 31,7\% sugeriría psicoterapia y sólo el 14,6\% menciona alguna terapia con nivel de evidencia I (psicoterapia cognitivoconductual o conductual); mientras que $38,8 \%$ de los residentes plantean psicoterapia, y sólo $8,3 \%$ terapias con nivel de evidencia I.

En relación a si el paciente requiere una interconsulta a otro servicio, el $80,5 \%$ de los internos y el $94,4 \%$ de los residentes lo consideran necesario ( $\mathrm{p}=0,052$, Chi2).

Las tablas 2 y 3 muestran el porcentaje de aciertos a cada una de las 10 preguntas sobre conocimientos para la elección de los antidepresivos. En la tabla 2 se comparan las respuestas de los internos y los residentes, y en la tabla 3 , las de los internos según la sede en que hicieron su externado en psiquiatría. Aquí podemos observar que la pregunta 3 (sobre fibromialgia), es la que tiene el mayor porcentaje de aciertos, con algo más de $60 \%$ para los internos y los residentes. Por otro lado, la de menor porcentaje de aciertos es la número 4 (sobre epilepsia), con un $17,1 \%$ de aciertos para los internos y un $27,8 \%$ para los residentes.

Tabla 2. Conocimientos sobre elección de los antidepresivos en internos y residentes de medicina

\begin{tabular}{lccc}
\hline Preguntas: & $\begin{array}{c}\text { Internos } \\
(\mathrm{n}=82)\end{array}$ & $\begin{array}{c}\text { Residentes } \\
(\mathrm{n}=36)\end{array}$ & $\mathrm{P}$ \\
\hline $\begin{array}{l}\text { 1: Si la paciente no quisiera aumentar de peso, ¿Cuál de los } \\
\text { siguientes escogería? }\end{array}$ & $20(24,4 \%)$ & $4(11,1 \%)$ & $0,049^{*}$ \\
$\begin{array}{l}\text { 2. Si la paciente tuviera un problema cardiaco (defecto de } \\
\text { conducción), ¿Cuál de los siguientes no le daría? }\end{array}$ & $37(45,1 \%)$ & $18(50 \%)$ & 0,688 \\
$\begin{array}{l}\text { 3. Si la paciente sufriera de fibromialgia, ¿Cuál de los siguientes } \\
\text { escogería? }\end{array}$ & $51(62,2 \%)$ & $22(61,1 \%)$ & 0,454 \\
$\begin{array}{l}\text { 4. Si la paciente tuviera epilepsia, ¿Cuál de los siguientes } \\
\text { preferiría? }\end{array}$ & $14(17,1 \%)$ & $10(27,8 \%)$ & 0,908 \\
$\begin{array}{l}\text { 5. Si la paciente tuviera Diabetes Mellitus, ¿Cuál sería el fármaco } \\
\text { menos recomendado? }\end{array}$ & $38(46,3 \%)$ & $10(27,8 \%)$ & $0,032^{*}$ \\
$\begin{array}{l}\text { 6. Si la paciente no desea tener efectos adversos en su vida sexual, } \\
\text { ¿Cuál escogería? }\end{array}$ & $39(47,6 \%)$ & $8(22,2 \%)$ & $0,005^{*}$ \\
$\begin{array}{l}\text { 7. Si la paciente tuviera retención urinaria, ¿Cuál de los siguientes } \\
\text { preferiría? }\end{array}$ & $17(20,7 \%)$ & $8(22,2 \%)$ & 0,573 \\
$\begin{array}{l}\text { 8. Si la paciente tuviera glaucoma de ángulo cerrado, ¿Cuál sería } \\
\text { el menos recomendable? }\end{array}$ & $38(46,3 \%)$ & $18(50 \%)$ & 0,644 \\
$\begin{array}{l}\text { 9. Si la paciente desea un fármaco con el menor efecto sedante o } \\
\text { hipnótico, ¿Cuál escogería? }\end{array}$ & $30(36,6 \%)$ & $6(16,7 \%)$ & $0,015^{*}$ \\
$\begin{array}{l}\text { 10. Si la paciente además tuviera trastorno de ansiedad } \\
\text { generalizada, ¿Qué antidepresivo no utilizaría? }\end{array}$ & $18(22 \%)$ & $8(22,2 \%)$ & 0,510 \\
\hline
\end{tabular}

*Diferencia estadísticamente significativa, para el análisis se utilizo la prueba de Chi2 
Tabla 3. Conocimientos sobre elección de los antidepresivos entre los internos

\begin{tabular}{|c|c|c|c|c|}
\hline \multirow[t]{2}{*}{ Preguntas: } & \multicolumn{2}{|c|}{ Internos } & \multirow[b]{2}{*}{ Total } & \multirow[b]{2}{*}{$\mathrm{p}$} \\
\hline & $\begin{array}{l}\text { INSM } \\
(\mathrm{n}=42)\end{array}$ & $\begin{array}{l}\mathrm{HNCH} \\
(\mathrm{n}=40)\end{array}$ & & \\
\hline $\begin{array}{l}\text { 1: Si la paciente no quisiera aumentar de peso, ¿Cuál de los } \\
\text { siguientes escogería? }\end{array}$ & $7(16,7 \%)$ & $13(32,5 \%)$ & $20 / 82$ & 0,106 \\
\hline $\begin{array}{l}\text { 2. Si la paciente tuviera un problema cardiaco (defecto de } \\
\text { conducción), ¿Cuál de los siguientes no le daría? }\end{array}$ & $20(47,6 \%)$ & $17(42,5 \%)$ & $37 / 82$ & 0,642 \\
\hline $\begin{array}{l}\text { 3. Si la paciente sufriera de fibromialgia, ¿Cuál de los } \\
\text { siguientes escogería? }\end{array}$ & $26(61,9 \%)$ & $25(62,5 \%)$ & $51 / 82$ & 0,955 \\
\hline $\begin{array}{l}\text { 4. Si la paciente tuviera epilepsia, ¿Cuál de los siguientes } \\
\text { preferiría? }\end{array}$ & $6(14,3 \%)$ & $8(20,0 \%)$ & $14 / 82$ & 0,493 \\
\hline $\begin{array}{l}\text { 5. Si la paciente tuviera Diabetes Mellitus, ¿Cuál sería el } \\
\text { fármaco menos recomendado? }\end{array}$ & $17(40,5 \%)$ & $21(52,5 \%)$ & $38 / 82$ & 0,276 \\
\hline $\begin{array}{l}\text { 6. Si la paciente no desea tener efectos adversos en su vida } \\
\text { sexual, ¿Cuál escogería? }\end{array}$ & $15(35,7 \%)$ & $24(60,0 \%)$ & $39 / 82$ & $0,027^{*}$ \\
\hline $\begin{array}{l}\text { 7. Si la paciente tuviera retención urinaria, ¿Cuál de los } \\
\text { siguientes preferiría? }\end{array}$ & $7(16,7 \%)$ & $10(25,0 \%)$ & $17 / 82$ & 0,354 \\
\hline $\begin{array}{l}\text { 8. Si la paciente tuviera glaucoma de ángulo cerrado, ¿Cuál } \\
\text { sería el menos recomendable? }\end{array}$ & $17(40,5 \%)$ & $21(52,5 \%)$ & $38 / 82$ & 0,276 \\
\hline $\begin{array}{l}\text { 9. Si la paciente desea un fármaco con el menor efecto sedante } \\
\text { o hipnótico, ¿Cuál escogería? }\end{array}$ & $13(31,0 \%)$ & $17(42,5 \%)$ & $30 / 82$ & 0,279 \\
\hline $\begin{array}{l}\text { 10. Si la paciente además tuviera trastorno de ansiedad } \\
\text { generalizada, ¿Qué antidepresivo no utilizaría? }\end{array}$ & $10(23,8 \%)$ & $8(20,0 \%)$ & $18 / 82$ & 0,677 \\
\hline
\end{tabular}

*Diferencia estadísticamente significativa, para el análisis se utilizo la prueba de Chi2

HNCH: Hospital Nacional Cayetano Heredia

INSM: Instituto Nacional de Salud Mental "Honorio Delgado - Hideyo Noguchi"

Considerando las 10 preguntas de opción múltiple, el promedio de respuestas acertadas en el grupo de los internos fue 3,8 $(\mathrm{DE}=1,99)$ mientras que entre los residentes, fue 3,08 $(\mathrm{DE}=1,56)(\mathrm{p}=0,097$, prueba de Mann-Whitney). Asimismo, el número de aciertos en estas preguntas fue significativamente mayor entre los internos que hicieron externado en psiquiatría en el $\mathrm{HNCH}[4,28(\mathrm{DE}=2,06)]$ respecto a los que hicieron dicha rotación en el INSM $[3,36(\mathrm{DE}=1,83)](\mathrm{p}=0,033$, prueba de Mann-Whitney).

\section{DISCUSIÓN}

Resulta satisfactorio que más del $90 \%$ de los residentes e internos realizaron un correcto diagnóstico de depresión mayor. A diferencia de los resultados encontrados en un trabajo sobre conocimientos de trastorno de pánico y trastorno de ansiedad generalizada, en donde se halló que sólo $70 \%$ y $22,9 \%$ de los médicos evaluados, respectivamente, los diagnosticaron correctamente (12).
Asimismo casi el $100 \%$ de los participantes consideró que el paciente requiere un tratamiento farmacológico, lo cual es correcto.

En cuanto al fármaco de primera línea, aproximadamente el $90 \%$ considera un ISRS, que según las guías de tratamiento es lo adecuado (13-16). Además se prefiere la sertralina y la fluoxetina, lo cual es coherente con el nivel socio-económico de los pacientes que acuden a instituciones de salud del estado.

Sin embargo podemos observar que existen deficiencias en cuanto a la elección de un tratamiento completo correcto (que incluye dosis y duración adecuadas), ya que sólo la mitad de los internos y un tercio de los residentes lo hacen. Algo similar encontramos en los resultados del trabajo de conocimientos sobre pánico y ansiedad generalizada, en donde casi el $90 \%$ de los participantes mencionan que el paciente requiere tratamiento farmacológico, pero se encuentra que sólo un $25 \%$ aproximadamente lo prescribe correctamente (12). Estudios en otras partes 
del mundo también encuentran deficiencias en cuanto a los conocimientos respecto a la dosis y la duración del tratamiento para la depresión. Por ejemplo, en un trabajo realizado en Estados Unidos más del 40\% de médicos no psiquiatras respondió incorrectamente cuando se les interrogó respecto a la duración del tratamiento (17); y en Ontario, Canadá, los médicos de atención primaria ante un anciano con depresión usarían dosis bajas del antidepresivo y más de la mitad retiraría prematuramente el fármaco (18).

Por otro lado es interesante ver que casi el 100\% de los residentes e internos refieren que agregaría además otra terapia a la farmacológica. Sin embargo, aunque una gran mayoría consideró la psicoterapia en general, el porcentaje de aquellos que indicaron una psicoterapia específica con nivel de evidencia I fue muy bajo.

Con la excepción de la pregunta relacionada con la fibromialgia, se encuentra que no más de la mitad de los internos y residentes aciertan las preguntas sobre cómo elegir los antidepresivos en función de las condiciones comórbidas y los efectos adversos del fármaco. No obstante, se observó una tendencia de los internos a obtener mejores respuestas que los residentes, probablemente debido a que su rotación por el servicio de psiquiatría había sido el año previo y recordaban con mayor claridad lo aprendido en la misma. Y dentro de los internos se ve que los que realizaron su rotación del externado de psiquiatría en el $\mathrm{HNCH}$ obtuvieron más respuestas acertadas que los que la realizaron en el INSM.

Dado que son los médicos generales los que en su mayoría evalúan inicialmente al paciente con depresión mayor, consideramos importante fortalecer los conocimientos acerca de la salud mental, para que en un futuro puedan realizar un adecuado manejo de estos pacientes $(7,9)$.

Dentro de las limitaciones del estudio, podría plantearse que algunas de las preguntas sobre elección de fármacos que se detallan en Materiales y Métodos son demasiado especializadas y no sería razonable esperar que internos y residentes de especialidades diferentes a la psiquiatría las respondan acertadamente. Asimismo, la pregunta 3 considera como respuesta correcta a la amitriptilina en base a que es el antidepresivo con evidencia más sólida de eficacia para la fibromialgia según lo referido en la última guía para el tratamiento de la depresión de la APA (13). Sin embargo, hay estudios que sugieren que otros antidepresivos tales como la nortriptilina (alternativa a) (19) y la venlafaxina (alternativa b) (20) podrían ser útiles.

Asimismo, es importante considerar que para llegar al diagnóstico de un trastorno mental no sólo es importante conocer los síntomas sino también es necesario tener las habilidades para explorar dichos síntomas en la entrevista y el examen mental, lo cual no se puede evaluar a través de una viñeta. Sin embargo, otros estudios para evaluar conocimientos para el diagnóstico de trastornos mentales se han realizado también con viñetas (21).

Otra observación es que hay que interpretar con cautela las diferencias encontradas entre los grupos que se muestran en las tablas 2 (internos vs. residentes) y 3 (internos que habían hecho externado de psiquiatría en el HNCH vs. los que lo habían hecho en el INSM), debido a que como se hacen muchas comparaciones algunas diferencias podrían haber ocurrido por simple azar, más aún en el caso de los p que son ligeramente inferiores a 0,05 .

Además, los resultados obtenidos en este estudio no se pueden extrapolar a todos los internos de otras universidades, ya que sólo los de la UPCH tienen rotación de externado en psiquiatría. Por otro lado, aproximadamente sólo la mitad de los residentes respondió la encuesta, lo cual hace que los resultados no se puedan generalizar a todos los residentes de la $\mathrm{UPCH}$.

A partir de los resultados obtenidos, nos parecería pertinente realizar nuevos proyectos que incluyan internos y residentes de otros hospitales y otras facultades. Por ejemplo, se podría evaluar los conocimientos luego de brindar capacitaciones o realizar comparaciones de la cantidad de diagnósticos psiquiátricos que se generan antes y después de las mismas.

Finalmente concluimos que, entre los individuos evaluados, la gran mayoría identifica el diagnóstico de depresión mayor, elijen un medicamento correcto y sugieren interconsultas razonables.

\section{Correspondencia}

Elisa Juárez Chávez

Calle Néstor Batanero 137 dpto. 302, Santiago de Surco, Lima. Perú.

Correo electrónico: elisa.juarez@upch.pe 


\section{REFERENCIAS BIBLIOGRÁFICAS}

1. Parikh SV, Lam RW, Kennedy SH, Enns MW, Kutcher $\mathrm{SP}$, Ravindran AV, et al. Clinical Guidelines for the Treatment of Depresive Desorders: I. Definitions, Prevalence and Health Burden. Can J Psychiatry 2001; 46 (Suppl. 1).

2. WHO: The global burden of disease 2004 update. Geneva: WHO Press, World Health Organization; 2008.

3. Instituto Nacional de Salud Mental. Estudio Epidemiológico Metropolitano de Salud Mental 2002. Anales de Salud Mental 2002; 18 (1-2).

4. Instituto Nacional de Salud Mental. Estudio Epidemiológico en Salud Mental en la Sierra Peruana 2003. Anales de Salud Mental 2004; 19 (1-2).

5. Instituto Nacional de Salud Mental. Estudio Epidemiológico de Salud Mental en la Selva Peruana 2004. Anales de Salud Mental 2005; 21(1-2).

6. Mojtabai R, Olfson M. Treatment seeking for depression in Canada and the United States. Psychiatr Serv 2006; 57:631-639.

7. De La Grecca R, Zapata M, Vega J, Mazzotti G. Manejo de pacientes con trastornos mentales en servicios ambulatorios de Medicina General en tres hospitales de Lima. Rev Med Hered 2005; 16 (4): 246 - 252.

8. Zapata-Vega MI, De la Grecca R, Altamirano A, Gonzaga V, Vega-Dienstmaier JM. Diagnosing depression: Symptoms and vocabulary used by a sample of general practitioners. Rev Neuropsiquiatr 2010;73:77-83.

9. De La Grecca R, Zapata M, Vega J, Mazzotti G. Patrones de tratamiento de pacientes con depresión en la consulta ambulatoria de medicina general. Rev Neuropsiquiatr 2005; 68(3-4): 182-190.

10. Paykel ES, Priest RG. Recognition and management of depression in general practice: consensus statement. BMJ 1992;305:1 198-202.

11. Escalante M, Uribe RM. Guía de Práctica Clínica en Depresión. Lima: Ministerio de Salud; 2008.

12. Changana-Arroyo A, Vega-Dienstmaier JM. Conocimientos acerca del trastorno de ansiedad generalizada y el trastorno de pánico con y sin agorafobia entre médicos residentes de medicina en entrenamiento durante el año 2009. Rev Neuropsiquiatr 2010; 73 (1): 2-8.

13. Gelenberg AJ, Freeman MP, Markowitz JC, Rosenbaum JF, Thase ME, Trivedi MH, et al. Practice Guideline for the Treatment of Mayor Depresive Disorder, 3rd Edition. Am J Psychiatry 2010;167 (10).

14. Bauer M, Bschor T, Pfennig A, Whybrow PC, Angst J, Versiani M, Moller H, et al. World Federation of Societies of Biological Psychiatry (WFSBP) Guidelines for Biological Treatment of Unipolar Depressive Disorders in Primary Care. World J Biol Psychiatry 2007; 8(2): 67-104.

15. Segal ZV, Kennedy DK, Lam RW, Enns MW, Kutcher $\mathrm{SP}$, Ravindran AV, et al. Clinical Guidelines for the Treatment of Depresive Desorders: III. Psychotherapy. Can J Psychiatry 2001; 46 (Suppl. 3).

16. Kennedy SH, Lam RW, Cohen NL, Ravindran AV, Enns MW, Kutcher SP, et al. Clinical Guidelines for the Treatment of Depresive Desorders: IV. Medication and other Biological Treatments. Can J Psychiatry 2001; 46 (Suppl. 4)

17. Shao WA, Williams JW Jr, Lee S, Badgett RG, Aaronson B, Cornell JE. Knowledge and attitudes about depression among non-generalists and generalists. J Fam Pract 1997;44(2):161-8.

18. Fitch K, Molnar FJ, Power B, Wilkins D, Man-SonHing M. Antidepressant use in older people: family physicians' knowledge, attitudes, and practices. Can Fam Physician 2005;51:80-1.

19. Heymann RE, Helfenstein M, Feldman D. A doubleblind, randomized, controlled study of amitriptyline, nortriptyline and placebo in patients with fibromyalgia. An analysis of outcome measures. Clin Exp Rheumatol 2001;19(6):697-702.

20. Sayar K, Aksu G, Ak I, Tosun M. Venlafaxine treatment of fibromyalgia. Ann Pharmacother 2003;37(11):1561-5.

21. Chen H, Parker G, Kua J, Jorm A, Loh J. Mental health literacy in Singapore: a comparative survey of psychiatrists and primary health professionals. Ann Acad Med Singapore 2000;29(4):467-73. 